Publ. RIMS, Kyoto Univ.

12 Suppl. (1976), 19-37.

\title{
Analytic Completion and Decomposability Properties in Tuboid Domains
}

by

J. BROS*

The analytic structure of distributions-i.e. the decomposability of the latter in sums of boundary values of analytic functions can be investigated not only from the microlocal point of view, but more globally in complex space. For this purpose we introduce in $\mathbb{C}^{n}$ (or in more general manifolds) a large class of domains $D$ called "tuboids", the closure of which contains a real domain $\Omega\left(\bar{\Omega}=\bar{D} \cap \boldsymbol{R}^{n}\right)$ :the boundary values on $\Omega$ of functions which are analytic in $D$ can then be considered (here in the sense of distributions). It turns out that the decomposability properties in domains of this type are intimately related with the geometry of the latter, in particular with their pseudoconvexity properties. In this connection, we shall state some pseudoconvexity criterions and theorems of analytic completion.

In the first part of this lecture, one is concerned with analytic functions in tuboids of a special kind, namely (respectively) tubes and local tubes in $\mathbb{C}^{n}$; these classes of analytic functions can be very well characterized by means of the (respectively) usual and generalized Fourier-Laplace transformation. In fact the geometry of the above domains in $\mathbb{C}^{n}$ will be exactly reflected by the corresponding geometry of "gauge-sets of exponential decrease" in the Fourier-space. Some theorems of analytic completion then appear as direct applications of this method: in the case of tubes, one reobtains the fact that the holomorphy envelope of a tube is its convex hull (Bochner's theorem); in the case of local tubes, a similar theorem has been derived; the edge-of-the-wedge theorem is a refinement

Received September 6, 1976.

* C. E. N. Saclay, France. 
of the latter. We then use the same method to prove a similar, but more general property of analytic completion, which we call "cut-tube theorem"; it applies to couples of analytic functions $\left(F^{+}, F^{-}\right)$in (respective) tubes $\left(T^{+}, T^{-}\right)$which are symmetric with respect to a certain analytic surface $\Sigma$, the discontinuity of $\left(F^{+}, F^{-}\right)$across $\Sigma$ being submitted to certain positivity conditions. A similar statement holds in the case of local tubes.

The second part of this lecture deals with general tuboids; a tuboid $D$ over a real set $\Omega$ is "microlocally" characterized by its "profile", which is a cone bundle $\Lambda=\bigcup_{p \in \Omega}\left(p, \Lambda_{p}\right)$ in the tangent bundle $T \Omega$ to $\Omega$. It turns out that the convexity of all (concical) fibers $\Lambda_{p}$ of $\Lambda$ is a characteristic property for the tuboids which are holomorphy domains. More precisely two statements can be proved: the one asserts that any tuboid $D$ with profile $\Lambda$ can be completed to another tuboid, whose profile $\hat{\Lambda}$ is obtained from $\Lambda$ by convex completion of all fibers of the latter; the other one asserts that if $\Lambda$ is a cone bundle with convex fibers, there exists a basis of tuboids with profile $\Lambda$ which are Stein manifolds. A special case of this result is the Cartan-Grauert theorem according to which every real domain $\Omega$ admits a basis of complex neighbourhoods which are Stein manifolds.

Our third part is devoted to a brief review of the decomposability properties which are consequences of the previous methods and results.

On the one hand, the (respectively) usual and generalized Laplace transform theorems, stated in part I, allow us to prove decomposition theorems for distributions in terms of boundary values of analytic functions in (respectively) tubes and local tubes. A microlocal (and therfore weaker) version of this decomposability property then follows, and leads in a natural way to the introduction of the "essential support" of a distribution in $\Omega$, as a certain closed subset of the cotangent bundle $T^{*} \Omega$; it establishes at once the "vertical decomposability" of the essential support (i.e. decomposability in each fiber of $T^{*} \Omega$ ). 
On the other hand, the geometrical properties of tuboids obtained in part II entail (as an application of Cartan's theorem B) a certain class of "horizontal decomposability properties" for functions which are analytic in tuboids.

These two types of decomposability ("vertical" and "horizontal") are the basic ingredients for proving the general decomposability of the essential support of a distribution*.

Finally a simple homological argument is briefly presented which relates every property of "support decomposability" (in a large sense) of distributions with a corresponding class of "generalized edge-of-thewedge theorems" for the associated analytic functions.

Remark. In all these results, the emphasis is intentionally put on the geometrical aspects; when boundary values are considered, they are always chosen to be distributions for convenience, but this is certainly not crucial.

\section{The Usual and Generalized Fourier-Laplace Transformation and Related Properties of Analytic Completion}

\section{$\S 1$. The Laplace Transform Theorem}

Notations: $f$ denotes a tempered distribution in $\mathbb{R}_{(x)}^{n}\left(x=\left(x_{1} \cdots x_{n}\right)\right)$ and $\tilde{f}=\mathscr{F} f$ its Fourier transform in $\boldsymbol{R}_{(p)}^{n}$; when it exists, we denote $F(k)=\frac{1}{(2 \pi)^{n / 2}} \int e^{i k . x} f(x) d x$ the Laplace transform of $f, F=\mathcal{L}(f)$, defined for $k=\left(k_{1}, \cdots, k_{n}\right)=p+i q$ complex (in $\left.\mathbb{C}^{n}(k)\right)$, and we then put $\tilde{f}=b . v \cdot F$ (b.v.= boundary value for $q \rightarrow 0)$.

\section{Gauge-sets of exponential decrease for $f$}

A closed star-shaped set $G$ in $\mathbb{R}_{(x)}^{n}$ is called a gauge-set of exponential decrease for a function $f$ if

* This property is analogous to the one which has been derived for hyperfunctions (from the flabbiness of the sheaf $\mathrm{C}$ ) in the Works by Professors M. Sato, T. Kawai, M. Kashiwara (see "Microfunctions and pseudo differential equations", Lecture Notes in Math., Springer-Verlag, 1971). 


$$
|f(x)| \leqq C(1+\|x\|)^{m} e^{-\frac{\|x\|}{r(u)}}
$$

for appropriate constant $C$ and integer $m$, where $\|x\|=\gamma(u)$ denotes the equation of the boundary of $G$ in polar coordinates $\left(x=\|x\| u, u \in \mathbb{S}^{n-1}\right)$.

In the case of a general tempered distribution $f, G$ is a gauge-set for $f$ if there exist polynomials $P$ and $Q$ and a bounded function $g$ satisfying (1) such that:

$$
f=P\left(\frac{\partial}{\partial x}\right)[Q(x) \cdot g(x)]
$$

One can prove:

Proposition 1. If $G_{1}, G_{2}$ are gauge-sets for $f$, then $G_{1} \cap G_{2}$ is also a gauge-set for $f$.

Reciprocal polar transformation between $\mathbb{R}_{(q)}^{n}$ and $\mathbb{R}_{(x)}^{n}$.

With every set $B$ in $\boldsymbol{R}_{(q)}^{n}$, we associate its polar set

$$
\widetilde{B}=\left\{x \in \boldsymbol{R}_{(x)}^{n} ; q \cdot x+1 \geqq 0, \forall_{q} \in B\right\},
$$

which is necessarily closed, convex and contains the origin of $\mathbb{R}_{(x)}^{n}$. Similarly $\widetilde{G}$ will denote the polar set in $\mathbb{R}_{(q)}^{n}$ of a subset $G$ of $\mathbb{R}_{(x)}^{n}$.

Proposition 2. The polar set of $G_{1} \cap G_{2}$ is $\left(\widetilde{G}_{1} \cup \widetilde{G}_{2}\right)^{c}$ (the notation $B^{c}$ denotes the convex hull of the set $B$ ).

Proposition 3. For set $B$ in $\mathbb{R}_{(q)}^{n}$, one has $\widetilde{\widetilde{B}}=(\bar{B} \cup\{0\})^{c}$.

Tubes in $\mathbb{C}_{(k)}^{n}$. We put $T_{B}=\mathbb{R}_{(p)}^{n}+i B\left(B \subset \mathbb{R}_{(q)}^{n}\right)$, the tube with basis $B$.

We shall always assume that the closure $\bar{B}$ of $B$ contains the origin.

Laplace transform theorem (L.T.T). The following statements hold:

i) $F$ analytic in $T_{B}$, continuous and slowly increasing at inf- 
nity in $T_{\bar{B}} \Rightarrow F=\mathcal{L}(f)$, and $\widetilde{B}$ is a gauge-set (of exponential decrease) for $f$.

ii) $f$ admits $G$ as a gauge-set $\Rightarrow F=\mathcal{L}(f)$ exists and is analytic and slowly increasing in $T_{B}$, where $B=\widetilde{G}$ (the interior of $\widetilde{G}$ ).

iii) If $B$ is a convex set, the following equivalence property holds: $F$ analytic and slowly increasing in $T_{B} \Leftrightarrow F=\mathcal{L}(f)$ and $\widetilde{B}$ is a gauge-set for $f$.

Remark. This result is still valid when $B$ is "flat" (i.e. contained in a linear submanifold): $F$ is then analytic only in the corresponding variables).

\section{§ 2. Bochner's Theorem, the Tubular Edge-of-the-Wedge and the Cut-Tube Theorem}

By applying successively the statements i) and ii) of the above Laplace transform theorem and noticing that $\widetilde{B}$ is the closed convex hull of $B$ (cf. proposition 3), one readily obtains:

Tube theorem. Every function which is analytic in a tube $T_{B}$ and slowly increasing at infinity can be analytically continued in the tube which is the convex hull of $T_{B}$ (it is also slowly increasing at infinity in the latter).

Remark. The general version of Bochner's tube theorem* (i.e. without conditions at infinity) will be also obtained as a consequence of the theorem on local tubes given below; in fact it will be seen that any tube can be considered as the union of an increasing sequence of local tubes of the considered type.

A refined version of the tube theorem is the

Tubular edge-of-the-wedge theorem.

Let $\tilde{f}=b . v \cdot F_{1}=b . v \cdot F_{2}$, with $F_{j}(j=1,2)$ analytic in the convex tube $T_{B_{j}}$; then $F_{1}, F_{2}$ have a common analytic continuation $F$ in $T_{B}$, where $B$ is the convex hull of $B_{1} \cup B_{2}$ (see fig. 1 ) and $\tilde{f}=b . v . F$.

\footnotetext{
* see S. Bochner, Annals of Math. 39, p. 14 (1938).
} 


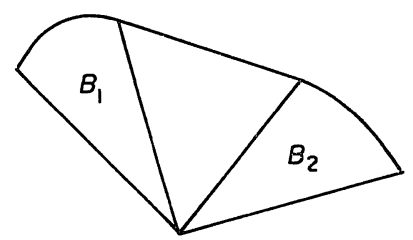

Fig. 1.

Proof. In view of L.T.T.(i), $f$ admits $\widetilde{B}_{1}$ and $\widetilde{B}_{2}$ as gauge-sets; in view of proposition 1 , it thus admits $\widetilde{B}_{1} \cap \widehat{B}_{2}$; then L.T.T. (ii) yields the desired result, inview of proposition 2 .

This result still holds in the case when $T_{B_{1}}, T_{B_{2}}$ are "flat tubes" (fig. 2); in this case $F_{1}, F_{2}$ are only analytic in certain variables, and are given as distributions with respect to the remaining variables; however their common analytic continuation $F$ is still defined and analytic in $T_{\left(B_{1} \cup B_{2}\right) c}$ (it is therfore analytic in more variables than the original $\left.F_{1}, F_{2}\right)$ : this result is known as the "flat tube theorem", and was first proved independently by Malgrange, Zerner, Kunze and Stein (1961).

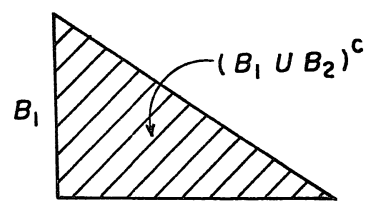

$B_{2}$

Fig. 2.

Analytic couples with a discontinuity of positive type.*

In $\boldsymbol{C}_{(w, \sigma)}^{r+n}\left(w \in \boldsymbol{C}^{r}, \sigma \in \boldsymbol{C}^{n}\right)$, we consider two open tubes $T_{B^{*}}=\boldsymbol{R}^{n+r}$ $+i B^{ \pm}$, with $B^{+}$and $B^{-}$symmetrical with respect to the surface $\{\operatorname{Im} \sigma=0\}$ (as in Fig. 3). We put: $B=\left(B^{+} \cup B^{-}\right)^{c}$,

$$
\begin{aligned}
& \Delta=B \cap\{\operatorname{Im} \sigma=0\}, \\
& \Delta^{+}=\left(B^{+} \cup \Delta\right)^{c} \\
& \Delta^{-}=\left(B^{-} \cup \Delta\right)^{c}
\end{aligned}
$$

* Properties of the same nature have also been derived independently by V. Glaser (unpublished), by using direct methods in complex place. 


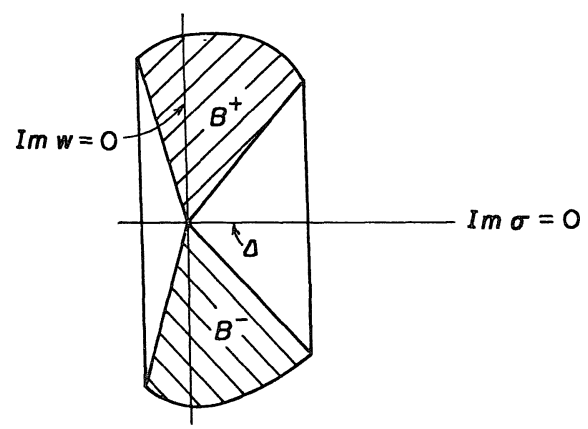

Fig. 3.

\section{Cut-tube theorem.}

Let $F^{+}, F^{-}$be analytic and slowly increasing functions in the respective tubes $T_{B^{+}}, T_{B^{-}}, \tilde{f}^{ \pm}=b \cdot v \cdot F^{ \pm}$and $\tilde{f}=\tilde{f}^{+}-\tilde{f}^{-}$.

Then, if the "discontinuity" $\tilde{f}$ is of positive type with respect to $w$ (i.e. if $\forall \varphi \in \mathscr{S}\left(\boldsymbol{R}_{(w)}^{r}\right), \forall \chi \in \mathscr{S}\left(\boldsymbol{R}_{(\sigma)}^{n}\right), \int \tilde{f}\left(w-w^{\prime}, \sigma\right) \chi(\sigma) \bar{\varphi}(w) \varphi\left(w^{\prime}\right)$ $\left.d w d w^{\prime} d \sigma \geqq 0\right), \tilde{f}$ admits an analytic continuation $F$ with respect to $w$ in the flat tube $T_{\Delta}$.

Moreover $F^{+}, F^{-}$can be analytically continued in the respective interiors of the tubes $T_{\Delta^{+}}, T_{\Delta^{-}}$, and in $T_{\Delta}$ one has:

$$
F=b . v \cdot F^{+}-b \cdot v \cdot F^{-} \text {. }
$$

Proof. We first treat the case when $F^{+}, F^{-}$are sufficiently decreasing at infinity, so that $f^{ \pm}=\mathscr{I}^{-1}\left(\tilde{f}^{ \pm}\right)$are functions which admit respectively $\widetilde{B}^{+}, \widetilde{B}^{-}$as gauge-sets in $\boldsymbol{R}_{(x, y)}^{n+r}\left(x=\left(x_{1}, \cdots, x_{r}\right)\right.$ and $y=\left(y_{1}, \cdots, y_{n}\right)$ denote the respective conjugate variables to $w=\left(w_{1}, \cdots, w_{r}\right)$ and $\left.\sigma=\left(\sigma_{1}, \cdots, \sigma_{n}\right)\right)$; thus $\widetilde{B}^{+} \cup \widetilde{B}^{-}$is a gauge-set for $f=f^{+}-f^{-}$.

We now introduce the partial inverse Fourier transform:

$$
g^{ \pm}(x, \sigma)=\frac{1}{(2 \pi)^{r / 2}} \int e^{-i w x} \tilde{f}^{ \pm}(w, \sigma) d w
$$

In view of the positivity assumption on $\tilde{f}$, we have:

$$
\forall \sigma, g(x, \sigma)=g^{+}(x, \sigma)-g^{-}(x, \sigma) \geqq 0,
$$

which entails the inequality:

$$
\forall y,|f(x, y)|=\left|\frac{1}{(2 \pi)^{n / 2}} \int e^{-i \sigma y} g(x, \sigma) d \sigma\right| \leqq f(x, 0)
$$


Using the definition of gauge-sets, we easily deduce from this inequality that the set $S$ which is the convex hull of the plane $\{x=0\}$ with the set $S_{1}=\left(\widetilde{B}^{+} \cup \widetilde{B}^{-}\right) \cap\{y=0\}$ is a gauge-set for $f$. Now the symmetry of $\widetilde{B}^{+}, \widetilde{B}^{-}$with respect to $\{y=0\}$ implies that $S_{1}=\left\{\widetilde{B}^{+} \cap \widetilde{B}^{-}\right\} \cap\{y=0\}$ and that $S=\left[\left(\widetilde{B}^{+} \cap \widetilde{B}^{-}\right) \cup\{x=0\}\right]^{c}$. Therefore its polar set is $\widetilde{S}=\left(\bar{B}^{+}\right.$ $\left.\cup \bar{B}^{-}\right)^{c} \cap\{\operatorname{Im} \sigma=0\}=\bar{\Delta}$, and L.T.T. then entails the existence of an analytic continuation $F$ of $\tilde{f}$ in $T_{\Delta}$.

By now writing $f^{+}=f+f^{-}$, we infer that $\widetilde{B}^{-} \cup S$ is a gauge-set for $f^{+}$; thus (from proposition 1$), \widetilde{B}^{+} \cap\left(\widetilde{B}^{-} \cup S\right)$ is a gauge-set too, but since it coincides with $\widetilde{B}^{+} \cap S$ (whose polar set is $\vec{\Delta}^{+}$), L.T.T. yields the announced result for $F^{+}$(a similar argument holds for $F^{-}$).

The general case is treated by putting $\tilde{h}^{ \pm}=e^{-w^{2}} \tilde{f}^{ \pm}$, since $\tilde{h}^{ \pm}$and $\tilde{h}=\tilde{h}^{+}-\tilde{h}^{-}$will then satisfy the conditions of the previous case (note that the product $e^{-w^{2}} \tilde{f}$ of two functions of positive type is of positive type).

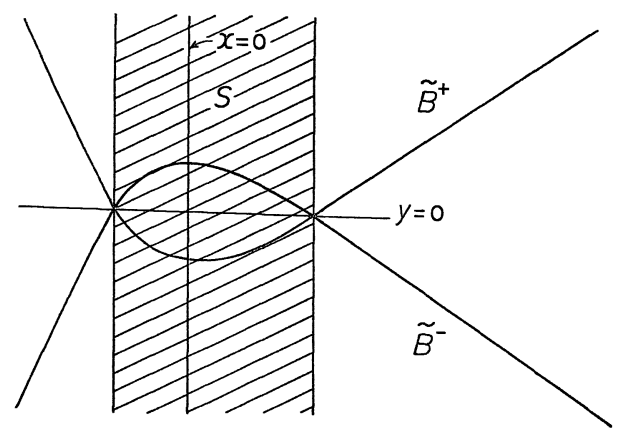

Fig. 4.

\section{§ 3. Generalized Laplace Transformation and Analytic Completion Theorems for Local Tubes}

$\Phi$ denotes an analytic function in $\mathbb{C}_{(k)}^{n}$, with a spherical critical point at $k=p_{0}(\mathrm{real})$, and such that: $\Phi(\bar{k})=\overline{\Phi(k)}$ (example: $\left.\Phi(k)=\sum_{j=1}^{n}\left(k_{j}-\left(p_{0}\right)_{j}\right)^{2}\right)$ A generalized Fourier transform $\mathscr{F}_{\oplus}$ associates with every function or distribution $f$ (with sufficient decrease at infinity) the following $n$-form in $\boldsymbol{R}_{\left(x, x_{0}\right)}^{n+1}\left(x=\left(x_{1} \cdots x_{n}\right)\right)$ :

$$
\mathscr{F}_{\varpi}(\tilde{f}) \equiv f\left(x, x_{0} ; p^{\prime}\right)=\sum_{j=0}^{n}(-1)^{j} f_{j}\left(x, x_{0} ; p^{\prime}\right) d x_{0} \wedge \cdots \wedge \widehat{d x_{j}} \wedge \cdots \wedge d x_{n}
$$


where

$$
\begin{aligned}
& f_{0}\left(x, x_{0}\right)=\frac{1}{(2 \pi)^{n / 2}} \int e^{-i p x-\varpi(p) x_{0}} \tilde{f}(p) d p \\
& \forall j 1 \leqq j \leqq n ; f_{j}\left(x, x_{0} ; p^{\prime}\right)=\frac{1}{(2 \pi)^{n / 2}} \int e^{-i p x-\varpi x_{0}} \rho_{j}\left(p ; p^{\prime}\right) \tilde{f}(p) d p
\end{aligned}
$$

here the analytic functions $\rho_{j}\left(k, k^{\prime}\right)$ are introduced through Hefer's theorem and satisfy:

$$
\Phi(k)-\Phi\left(k^{\prime}\right)=\sum_{j=1}^{n} \rho_{j}\left(k, k^{\prime}\right)\left(k_{j}-k_{j}^{\prime}\right) .
$$

Let $f(x)$ be the usual inverse Fourier transform of $\tilde{f}$; we note that $\left.\boldsymbol{f}\right|_{x_{0}=0}=f(x) d x_{1} \wedge \cdots \wedge d x_{n}$.

\section{Local tubes $T_{B \oplus \alpha}$.}

$\mathrm{T}_{B \varpi \alpha}$ is a domain in $\mathbb{C}_{(k)}^{n}$, defined as the bounded connected component of the following set:

$$
\bigcup_{b=(\rho, \omega) \in B}\left\{k ; \begin{array}{l}
\|q\|+\rho(\operatorname{Re} \Phi(p+i q)-\alpha)=0 \\
q=\|q\| \cdot \omega ; \rho>0 ; \omega \in \mathbb{S}^{n-1}
\end{array}\right\}
$$

where $(\rho, \omega)$ denote the polar coordinates of a point $b$ in an auxiliary space $\mathbb{R}_{(b)}^{n} ; B$ is an open set in this space, such that $0 \in \bar{B}$; it also has to be contained in a certain critical domain $B_{c r}$, determined by $\Phi$.

Remarks. i) The intersection of $\bar{T}_{B \varpi \alpha}$ with the real subspace is the closure of the open set $\Omega_{\alpha}=\left\{p \in \mathbb{R}^{n} ; 0 \leqq \Phi(p)<\alpha\right\}$ At every point $p$ in $\Omega_{\alpha}$, the profile of the corresponding imaginary section of $T_{B \varpi \alpha}$ (in $\mathbb{R}_{(q)}^{n}$ ) is given by the profile of $B$ in $\mathbb{R}_{(b)}^{n}$ (by "profile" of a domain, we always mean the cone of all semi-straight lines which are issued from the origin and whose intersection with the considered domain cantains a segment ending at the origin:

ii) For $\Phi=0, T_{B \varpi \alpha}$ reduces to the tube $T_{(\alpha B)}$ whose basis is the homotetic of $B$ with respect to the origin, the ratio being equal to $\alpha$.

\section{The $\alpha$-gauge-sets}

A cone $\Gamma$ with apex at the origin in the half-space $\left\{x_{0} \geqq 0\right\}$ of $\mathbb{R}_{\left(x, x_{0}\right)}^{n+1}$ 
is called an $\alpha$-gauge-set of exponential decrease for a function $f\left(x, x_{0}\right)$ if the latter satisfies bounds of the following type:

$$
\forall \varepsilon>0,\left|f\left(x, x_{0}\right)\right| \leqq C_{\varepsilon}\left(1+\|x\|^{m}\right) e^{-\alpha(1-\varepsilon) x_{0}}
$$

in the region $\left\{\left(x, x_{0}\right) \notin \Gamma ; x_{0} \geqq 0\right\}$. The same definition holds for a form $\boldsymbol{f}$ by requiring that all the coefficients $f_{j}$ of $\boldsymbol{f}$ satisfy the above inequality (uniformly with respect to the extra-variables $p^{\prime}$ ). Any such cone $\Gamma$ is called "admissible" if it contains the cone $\Gamma_{B_{c r}}$ with basis $\widetilde{B}_{c r}$ in $\left\{x_{0}=1\right\}$ and apex at the origin in $\boldsymbol{R}_{\left(x, x_{0}\right)}^{n+1}$.

The following property can then be proved:

Generalized Laplace Transform Theorem (G.L.T.T.). i) If the restriction of the distribution $f$ to $\Omega_{\alpha}$ is the boundary value of an analytic function $F$ defined in $T_{B \Phi \alpha}$, then the cone $\Gamma_{B}$ with basis $\widehat{B}$ in $\left\{x_{0}=1\right\}$ and apex at the origin in $\boldsymbol{R}_{\left(x, x_{0}\right)}^{n+1}$ is an $\alpha$-gauge-set for $f$

ii) If $\tilde{f}$ has an $\mathscr{F}$-transform $\boldsymbol{f}$ which admits an admissible $\alpha$-gauge-set $\Gamma$ then $\left.\tilde{f}\right|_{\Omega_{\alpha}}=b . v \cdot F$, where $F$ is an analytic function in the tube $T_{\widetilde{G}}^{\circ}$; here $G$ denotes the section of $\Gamma$ by the hyperplane $\left\{x_{0}=1\right\}$, and $\stackrel{\odot}{G}$ is the interior of the polar set of $G$.

iii) If $B$ is convex (and $B \subset B_{c r}$ ): $\left.\tilde{f}\right|_{\Omega_{\alpha}}=b . v \cdot F$, analytic in $T_{B \oplus \alpha}$ $\Leftrightarrow S_{B}$ is an $\alpha$-gauge-set for $\boldsymbol{f}$.

As in the case of the usual Laplace transformation, the following theorems of analytic completion are consequences of G.L.T.T.

Local tube theorem.* The envelope of holomorphy of a local tube $T_{B \oplus \alpha}$ (with $B \subset B_{c r}$ ) is the local tube $T_{B c \oplus \alpha}, B^{c}$ being the convex hull of $B$ in $\boldsymbol{R}_{(b)}^{n}$.

(Local) edge-of-the-wedge theorem.** Let $B_{1}, B_{2}$ touch each

* Microlocal analogues of this theorem have been proved independently by Professor Komatsu (J. Fac. Sci. Univ. Tokyo., IA. 19 (1972)) and E. Andronikof (Thesis, 1975, Paris).

** In its original microlocal version (not formulated with the $T_{\boldsymbol{B} \propto \alpha}$ ) this theorem has been proved by H. Epstein, J. Math. Phys. 1 (1969), 524. 
other at the origin in $\boldsymbol{R}_{(b)}^{n}$ (as in Fig. 1) and assume that $\left.\tilde{f}\right|_{\Omega_{\alpha}}=b \cdot v \cdot F_{1}$ $=b . v . F_{2}$, where $F_{1}, F_{2}$ are respectively analytic in, $T_{B 1 \omega \alpha}, T_{B 2 \oplus \alpha}$. Then $\left.\tilde{f}\right|_{s_{\alpha}}=b . v . F$, where $F$ is a common analytic continuation of $F_{1}$ and $F_{2}$ in $T_{B \varpi \alpha}$, with $B=\left(B_{1} \cup B_{2}\right)^{c}$.

A similar version of the flat tube theorem can be obtained. We also have:

\section{Semi-local version of the cut-tube theorem.}

$\mathbb{C}_{(k)}^{n}$ is again replaced by $\mathbb{C}_{(w, \sigma)}^{++n}$, but our localizing function $\Phi$ will only depend on the $n$ variables $\sigma$, and not on $w . B^{+}, B^{-}$are then given in a space $\boldsymbol{R}_{(I m w, b)}^{r+n}$ (as in Fig. 3, but with $\operatorname{Im} \sigma$ replaced by the auxiliary variables $\left.b=\left(b_{1}, \cdots, b_{n}\right)\right)$. The corresponding (semi)-local tubes $T_{B^{\star} \Phi \alpha}$ will then be bounded by the real open sets

$$
\left\{(w, \sigma) ; w \in \mathbb{R}^{n}, \sigma \in \Omega_{\alpha}(i . e .0 \leqq \Phi(\sigma)<\alpha)\right\}
$$

Our statement is then the following:

Proposition. Let $\tilde{f}^{ \pm}=$b.v. $F^{ \pm}$, with $F^{+}, F^{-}$respectively analytic in $T_{B^{+} \Phi \alpha}, T_{B^{-} \omega_{\alpha}}$ and assume $\tilde{f}=\tilde{f}^{+}-\tilde{f}^{-}$is of positive type with respect to w (for $\sigma$ varying in $\Omega_{\alpha}$ ). Then $F^{+}, F^{-}, \tilde{f}$ admit analytic continuations in the respective (semi)-local tubes $T_{\Delta^{+} \Phi \alpha}, T_{\Delta-\varpi \alpha}, T_{\Delta \varpi \alpha}$, with $\Delta^{+}$, $\Delta^{-}, \Delta$ defined as in paragraph 2.

\section{Tuboids and a Generalizalion of Cartan-Grauert's Theorem}

In $\boldsymbol{C}_{(k)}^{n}=\mathbb{R}_{(p)}^{n} \times \mathbb{R}_{(q)}^{n}$, identified with the tangent fiber bundle $T \mathbb{R}_{(p)}^{n}$ -or more generally in $T R, \mathscr{R}$ being an $n$-dimensional real analytic manifold-we introduce the following class of domains, called "profiles".

Definition 1. A profile in $T \mathscr{R}$ is an open "cone boundle" $\Lambda=\bigcup_{p \in \Omega}(p$, $\Lambda_{p}$ ); for every $p$ in the open set $\Omega$ of $R, \Lambda_{p}$ is a non-empty open cone with apex at the origin in $T_{p} R$.

We can then introduce the following class of domains, called "tuboids" in $\mathbb{C}_{(r)}^{n}$ or in a complex analytic manifold $\mathcal{M}$, which admits $\mathscr{R}$ as 
its real submanifold.

Definition 2. In $\mathbb{C}^{n}$, a tuboid $D$ with profile $\Lambda$ above the real open set $\Omega$ is a complex domain which satisfies the following properties:

$$
\forall \Lambda^{\prime}, \quad \text { with } \bar{\Lambda} \backslash \boldsymbol{R}^{n} \subset \Lambda^{\prime}, \exists V,
$$

a complex naighbourhood of $\Omega$, such that: $D \cap V \subset \Lambda^{\prime}$,

b)

$$
\forall \Lambda^{\prime \prime}, \text { with } \bar{\Lambda}^{\prime \prime} \backslash \boldsymbol{R}^{n} \subset \Lambda, \exists W,
$$

a complex neighbourhood of $\Omega$, such that: $\Lambda^{\prime \prime} \cap W \subset D$.

The situation is illustrated on Fig. 5 for each fiber $D_{p}\left(D=\bigcup_{p=\Omega}\left(p, D_{p}\right)\right)$ : $\Lambda_{p}$ is also called the profile of the fiber $D_{p}$.

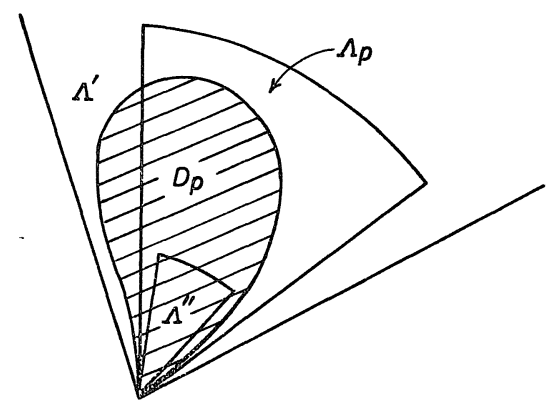

Fig. 5.

In a general analytic manifold $\mathscr{M}$, with real submanifold, $\mathscr{R}$ a tuboid $D$, with profile $\Lambda$ in $T \mathscr{R}$, is defined by requiring that, in a fixed (but arbitrary) covering of $\mathscr{M}$, its restriction to every chart is a tuboid in the corresponding system of local coordinates. As a matter of fact the above definition is invariant under all analytic changes of variables on $\mathscr{M}$.

Remark. Every local tube $T_{B \emptyset_{\alpha}}$ (introduced in I.3) is a tuboid in $C^{n}$ whose profile is of the form $A=\Omega \times \mathscr{C}, \mathscr{C}$ being the profile of $B$ in $\boldsymbol{R}_{(b)}^{n}$.

For every profile $\Lambda$ in $T \mathscr{R}$ we define a new profile $\hat{\Lambda}$ which is called the "convex hull of $\Lambda$ with respect to the fibers": if $\Lambda=\bigcup_{p \in g}\left(p, \Lambda_{p}\right)$, then $\hat{\Lambda}=\bigcup_{p \in \Omega}\left(p, \hat{\Lambda}_{p}\right)$ where $\hat{\Lambda}_{p}$ is the (usual) convex hull of $\hat{\Lambda}_{p}$. When $\Lambda=\hat{\Lambda}$, it is said to be "convex with respect to the fibers". 
We can now state the following properties of holomorphic convexity of the tuboids.

Theorem 1. For every tuboid $D$ with profile $\Lambda$, there exists a tuboid $\widehat{D}$ with profile $\widehat{\Lambda}$ such that every function $f$ which is analytic in $D$ can be analytically continued in $\widehat{D}$.

Theorem 2. For every tuboid $D$ whose profile $\Lambda$ is convex with respect to the fibers, there exists a tuboid $D^{\prime}$ contained in $D$, which has the same profile $\Lambda$ and is a Stein manifold.

In the case of local tubes $T_{B \omega \alpha}$, the statement of theorem 1 is a corollary of the "local tube theorem" given in I.3; the general case of theorem 1 is then treated by noticing that every tuboid $D$ with profile $\Lambda$ can be approximated, in the neighbourhood of each real point $p$ of its closure, by an infinite sequence of local tubes whose profiles (at $p$ ) tend to $\Lambda_{p}$.

The proof of theorem 2 contains the following steps: in $\mathbb{C}^{n}$, one first constructs a tuboid $D^{\prime \prime} \subset D$, which has the same profile $A$ as $D$, and whose all fibers $D_{p}^{\prime \prime}$ are convex open sets. Then, it is possible to construct a tuboid $D^{\prime} \subset D^{\prime \prime}$, with profile $\Lambda$, which is a holomorphy domain; the latter property is ensured by the definition of $D^{\prime}$ as being (a connected component of) the intersection $E\left(D^{\prime \prime}\right)$ of an appropriate family of holomorphy domains: each domain of this family is the complement in $C^{n}$ of a complex hypersphere $S_{a \eta \rho}$ :

$$
S_{a \eta \rho}=\left\{k \in \mathbb{C}^{n} ; \sum_{j=1}^{n} \eta_{j}\left(k_{j}-a_{j}+i \rho \eta_{j}\right)+i \sum_{j=1}^{n}\left(k_{j}-a_{j}+i \eta_{j}\right)^{2}=0\right\}
$$

(where $\left.a=\left(a_{1} \cdots a_{n}\right) \in \mathbb{R}^{n}, \eta=\left(\eta_{1} \cdots \eta_{n}\right) \in S^{n-1} ; 0 \leqq \rho \leqq \frac{1}{2}\right) . \quad E\left(D^{\prime \prime}\right)$ is defined as follows:

$$
E\left(D^{\prime \prime}\right)=\bigcap_{\substack{(\rho, \eta) \in \tilde{D}_{a^{\prime \prime}} \\ a \in \boldsymbol{R}^{n}}}\left(\mathrm{C} S_{a \eta \rho}\right) \cap \Theta
$$

Here $\widetilde{D}_{a}^{\prime \prime}$ denotes the polar set in $\boldsymbol{R}^{n}$ (with polar coordinates $\rho, \eta$ ) of the fiber $D_{a}^{\prime \prime}$ of $D$ at a. $\Theta$ is the tube: $\left\{k ;\|\operatorname{Im} k\|<\frac{1}{2}\right\}$. 
The case of a tuboid $D$ in a general $n$-dimensional manifold $\mathcal{M}$ is treated by making a regular imbedding " $i$ " of $\mathcal{M}$ in a complex space $C^{k}(k \geqq n)$; then $i(D)$ is exhibited as the restriction to $i(\mathscr{M})$ of a certain tuboid $\mathscr{D}$ in the ambient space $C^{k}$, to which the above construction of the corresponding tuboid $\mathscr{D}^{\prime}$ is applied: the section of this holomorphy domain $\mathscr{D}^{\prime}$ with the closed submanifold $i(\mathscr{M})$ is a Stein manifold which satisfies the required properties (it is a tuboid in $i(\mathcal{M})$ which is contained in $i(D)$, and has the same profile as the latter).

Remark. In the case when $\Lambda=\Omega \times \boldsymbol{R}^{n}$, a tuboid $D$ with profile $\Lambda$ is simply a complex neighbourhood of $\Omega$. In this case, theorem 2 reduces to Cartan-Grauert's theorem* according to which every complex neighbourhood of $\Omega$ contains a "Stein neighbourhood" of $\Omega$.

"Horizontal" decomposability properties.

By applying Cartan's theorem $B^{* *}$ (or the corresponding theorem due to Hörmander*** in the case of slowly increasing functions), one can derive from theorem 2 the two following decomposability properties. Notations: The profile $\Lambda=\bigcup_{p \in \Omega}\left(p, \Lambda_{p}\right)$ is said to be "above the real open set $\Omega$; if $\Omega^{\prime} \subset \Omega$, we put:

$$
\Lambda_{\Omega^{\prime}}=\bigcup_{p \in \Omega^{\prime}}\left(p, \Lambda_{p}\right)
$$

Theorem 3. Let $\left\{\Lambda_{j}\right.$ above $\left.\Omega_{j} ; j \in J\right\}$ be a finite family of profiles which are convex with respect to the fibers and such that:

$$
\forall i, j \in J,\left.\Lambda_{i}\right|_{\Omega_{i j}}=\left.\Lambda_{j}\right|_{\Omega_{i j}}\left(\text { with } \Omega_{i j}=\Omega_{i} \cap \Omega_{j}\right)
$$

Then for every family of functions $\left\{h_{i j} ; i, j \in J\right\}$ analytic in respective tuboids $D_{i j}$ with profiles $\Lambda_{i j}$, and satisfying the conditions:

$$
h_{i j}=-h_{i j}, h_{i j}+h_{i k}+h_{k i}=0 \text { in } D_{i j} \cap D_{j k} \cap D_{h i}
$$

there exists a family of functions $\left\{h_{i} ; i \in J\right\}$, analytic in tuboids $D_{i}$ with profiles $\Lambda_{i}$, such that

$$
h_{i j}=h_{i}-h_{j} \text { in } D_{i} \cap D_{j}
$$

* H. Cartan, Bull. Soc. Math. France, 85 (1957), 77.

H. Grauert, Ann. Math., Série 2, 68 (1958), 460.

** H. Cartan, Séminaires E. N. S., Paris (1951/52).

*** L. Hörmander, An introduction to complex analysis in several variables, Van Nostrand, Princeton (1966). 
(the $D_{j}$ 's only depend on the $D_{i j}$ 's and not on the functions $h_{i j}$ ).

Theorem 4. Let $\omega, \Omega$ be two real open sets such that $\omega \subset \Omega$, and $\Lambda$ a profile above $\Omega$ (convex with respect to the fibers).

For every function $h$, analytic in a tuboid $\Delta$ with profile $\Lambda \mid \omega$ there exists a decomposition $h=H+r$, where $H$ is analytic in a tuboid $D$ with profile $\Lambda$, and $r$ is analytic in a complex neighbourhood W of $\omega$ ( $D$ and $W$ only depend on $\Delta)$.

Corresponding versions of theorems 3 and 4 can be written in the case of functions with slow increase; in this case all functions have distribution boundary values in the corresponding real open sets $\Omega_{j}, \Omega, \omega$. Calling $\tilde{f}_{i}, \tilde{f}$ the b.v. of $h_{i}, h$, the above statements appear as certain decomposability properties of distributions; these properties are called "horizontal", since the terms of the decompositions are distributions defined in different open sets (see for example in theorem 4):

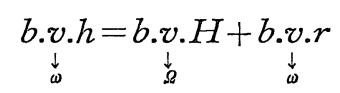

\section{Vanous Notions of Essential Support, Decomposability Prop- erties and Generalized Edge-of-the-Wedge Theorems}

\section{$\S 1$. Various Notions of "Essential Support" for Distributions}

The L.T.T. and G.L.T.T. (see I) have allowed us to characterize certain analyticity properties (in tubes and local tubes) by means of the corresponding properties of exponential decrease of the appropriate (Fourier of $\mathscr{F}_{\oplus}$ ) transform: there the geometrical notion of gauge-set of exponential decrease played an important role. More generally, we can introduce successively the following notions:

Tubular essential support $S_{t u b}(\tilde{f})$ of a tempered distribution $\tilde{f}$ in $\boldsymbol{R}_{(p)}^{n} . \quad S_{t u b}(\tilde{f})$ is the intersection of all the gauge-sets for the (inverse) Fourier transform of $\tilde{f}$; it is a closed star-shaped subset of $\mathbb{R}_{(x)}^{n}$.

Local essential support $S_{\alpha, \oplus}(\tilde{f})$ of $\tilde{f} . \quad S_{\alpha, \oplus}(\tilde{f})$ is the intersection 


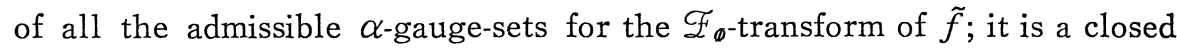
cone with apex at the origin in $\boldsymbol{R}_{\left(x, x_{0}\right)}^{(n+1)} \cap\left\{x_{0} \geqq 0\right\}$.

Remark. For every $\tilde{f}$ one can always construct a decreasing sequence (by inclusion) of gauge-sets (resp. $\alpha$-gauge-sets) which is a basis of closed neighbourhoods of $S_{t u b}(\tilde{f})$ (resp. $S_{\alpha, \oplus}(\tilde{f})$ ).

(Microlocal) essential support $S_{p}(\tilde{f})$ of $\tilde{f}$ at the point $P . S_{p}(\tilde{f})$ is the projective limit, for $\alpha \rightarrow 0$, of the family of cones $S_{\alpha, \Phi}(\tilde{f}) \cap\left\{x_{0}=0\right\}$ : it is a closed cone in $\boldsymbol{R}_{(x)}^{n}$ (or more generally in the fiber $T_{p} * \mathcal{R}$ of the cotangent boundle $T^{*} \mathscr{R}$ to the real manifold $\mathscr{R}$ where $\tilde{f}$ is defined). Its definition turns out to be independent of the choice of $\Phi$.

Essential support $S(\tilde{f})$ of a distribution $\tilde{f}$ in $\mathscr{R}$. $S(\tilde{f})$ is a closed cone bundle in $T^{*} \mathscr{R}$ which is defined by:

$$
S(\tilde{f})=\bigcup_{P \in \Xi}\left(p, S_{p}(\tilde{f})\right)
$$

\section{$\S 2$. Some Vertical Decomposability Properties of a Distribution}

With each notion of essential support (given above) will correspond a decomposability property of the distribution $\tilde{f}$ as a sum of boundary values of analytic functions in relevant tuboid domains. If $\mathcal{R}$ is the real manifold in which $f$ is given, all the involved tuboids have their profiles above $\mathscr{R}$ : this accounts for our terminology ("vertical" decomposability).

Tubular decomposability of a tempered distribution $\tilde{f}$ in $\boldsymbol{R}_{(p)}^{n}$. For every finite family of open convex sets $G_{i}(i \in I)$ containing the origin in $\boldsymbol{R}_{(x)}^{n}$, the condition

$$
S_{t u b}(\tilde{f}) \subset \bigcup_{i \in I} G_{i}
$$

implies the existence of a (non unique) decomposition: $\tilde{f}=\sum_{i \in I} \tilde{f}_{i}$

$$
\text { with } S_{t u b} \cdot\left(\tilde{f}_{i}\right) \subset G_{i}
$$

From L.T.T., $\tilde{f}_{i}=$ b.v.F $F_{i}$, analytic in the tube $T_{\widetilde{\sigma}_{i}}^{\circ}$. 
Decomposability of $\tilde{\boldsymbol{f}}$ in local tubes $\boldsymbol{T}_{\boldsymbol{B} \sigma \alpha}$ For every finite family of admissible open convex cones $\Gamma_{i}(i \in I)$ in the halfspace $\left\{x_{0} \geqq 0\right\}$ of $\boldsymbol{R}_{\left(x, x_{0}\right)}^{n+1}$, the condition $S_{\alpha, \Theta}(\tilde{f}) \subset\left(\bigcup_{i \in I} \Gamma_{i}\right) \cup\{0\}$ implies the existence of a (non-unique) decomposition: $\tilde{f}=\sum_{i \in I} \tilde{f}_{i}$, with $S_{\alpha, \Theta}\left(\tilde{f}_{i}\right) \subset \Gamma_{i} \cup\{0\}$ If the $\Gamma_{i}$ are salient, this is equivalent (from G.L.T.T.) to: $\tilde{f}_{i}=$ b.v.F. $F_{i}$, analytic in $T_{B_{i} \omega \alpha}$, ( $B_{i}$ being the interior of the polar set of $\Gamma_{i} \cap\left\{x_{0}=1\right\}$ ).

The idea of the proof of the latter property consists in the possibility of cutting into several pieces (contained in $\Gamma_{i}$ ) the $n$-dimensional manifold in $\boldsymbol{R}_{\left(x, x_{0}\right)}^{n+1}$ over which the inverse $\mathscr{F}_{\emptyset}$-formula is integrated. By a process of projective limit $(\alpha \rightarrow 0)$ one also obtains:

Microlocal decomposability of $\tilde{f}$ with respect to the fibers. Let $P$ be an arbitrary fixed point in $\boldsymbol{R}_{(p)}^{n}$ (or $\left.\mathcal{R}\right)$. For every finite family of open salient convex cones $\mathscr{C}_{i}(i \in I)$ in $\boldsymbol{R}_{(x)}^{n}$ (or $\left.T_{p} * \mathcal{R}\right)$ such that $S_{p}(\tilde{f}) \subset\left(\bigcup_{i \in I} \mathscr{C}_{i}\right) \cup\{0\}$, there exists a (non-unique) decomposition $\tilde{f}=\sum_{i \in I} \tilde{f}_{i}$ with: $\forall i \in I, S_{p}\left(\tilde{f}_{i}\right) \subset \mathscr{C}_{i} \cup\{0\}$

\section{$\S 3$. Generalized Edge-of-the-Wedge Theorems}

With every decomposability property of the previous type is associated a certain class of "generalized edge-of-the-wedge theorems", which correspond to the nullity of certain homology groups.

In fact, one can check that all the above notions of essential supports of distributions are special cases of the following algebraic notion:

"Support structure" on a vector-space $E$ with value in a set $F$. It is defined by giving a mapping $s$ from $E$ to $\mathscr{Q}(F)$ and a subset $\mathscr{Q}^{\prime}(F)$ of $\mathscr{P}(F)$ which is a $\sigma$-algebra, such that:

a)

$$
\begin{aligned}
& \forall f_{1}, f_{2} \in E, \quad \forall \lambda_{1}, \lambda_{2} \in C, \\
& s\left(\lambda_{1} f_{1}+\lambda_{2} f_{2}\right) \subset s\left(f_{1}\right) \cup s\left(f_{2}\right)
\end{aligned}
$$

b) decomposability property: for every finite family of sets $\gamma_{i}(i \in I)$ in $\mathscr{Q}^{\prime}(F)$, the condition $s(f) \subset \bigcup_{i \in I} \gamma_{i}$ implies the existence of a decomposition: $f=\sum_{i \in I} f_{i}$ with $s\left(f_{i}\right) \subset \gamma_{i}$. Such a support structure will be denoted $\left(E, F ; s, \mathscr{Q}^{\prime}(F)\right)$. 
In the above cases, $E$ is the space of distributions, in $\boldsymbol{R}_{(p)}^{n}, F$ is $\boldsymbol{R}_{(x)}^{n}$ (resp. $\left.\boldsymbol{R}_{\left(x, x_{0}\right)}^{n+1}\right), \mathscr{F}^{\prime}(F)$ is the set of open convex subsets containing 0 (resp. open salient convex cones with apex at the origin in the half-space $\left.\left\{x_{0} \geqq 0\right\}\right)$.

$\left(E, F ; s, \mathscr{Q}^{\prime}(F)\right)$ being given, consider a finite family $\mathscr{F}=\left\{\gamma_{i} \in\right.$ $\left.\mathscr{Q}^{\prime}(F) ; i \in I\right\}$ and put $N=|I|$ (the legth of the set $I$ ).

With $\mathscr{F}$, one can associate a certain homological complex $\mathcal{K}_{\Phi}$ $=\bigoplus_{p=0}^{N} \mathcal{K}_{\Phi}{ }^{(p)} ;$ the $p$-chains are sets

$$
\begin{aligned}
f^{(p)}=\left\{f_{J, \sigma_{J}}^{(p)}=\right. & \varepsilon_{\sigma_{J}} f_{J}^{(p)}, \text { with } f_{J}^{(p)} \in E, s\left(f_{J}^{(p)}\right) \subset \gamma_{J}, \\
& \forall J \subset I \text { with }|J|=p\}
\end{aligned}
$$

(here we have put $\gamma_{J}=\bigcup_{i \in J} \gamma_{i}, \sigma_{J}$ refers to an arbitrary permutation of the elements $J$, and $\varepsilon_{\sigma_{J}}$ is the signature of this permutation).

The operation $\delta$ (such that $\delta \circ \delta=0$ ) in $\mathcal{K}$ is defined by: $g^{(p-1)}=\delta f^{(p)}$ with: $g_{L, \tau_{L}}^{(p-1)}=\sum_{j \Subset L} f_{L \cup\{j\}, \tau_{L^{\prime}}}^{(p)}, \tau_{L}^{\prime}$ being the permutation of $L \cup\{j\}$ obtained from $\tau_{L}$ by adding the element $j$ on the right of $L$.

One can then prove:

Theorem. The homology groups $H_{p}$ of $\mathcal{K}_{I}$ all vanish for $1 \leqq p$ $\leqq N$.

In the applications to the various essential supports listed above, the $f^{(p)}$ appear as sets of b.v. of analytic functions $F_{J}^{(p)}$ in domains of the corresponding type $D_{J}^{(p)}$ (tubes, local tubes $T_{B \Phi \alpha}$, microlocal tubes centered at $P$ ). $\quad D_{J}^{(p)}$ is the holomorphy envelope (known explicitly) of the union of $p$ elementary domains $D_{j}(j \in J)$.

A typical example of the theorems thus obtained is the following (we choose $s=S_{\alpha, \mathscr{\Phi}}$, and write the vanishing of the group $H_{1}$ ):

Generalized eage-of-the-wedge theorem.* For every family of distributions $\tilde{f}_{j}=b . v \cdot F_{j}(j \in J)$, analytic in $T_{B_{j} \varpi \alpha}\left(B_{i}\right.$ convex $)$, and such

* Other versions of this theorem have been obtained by A. Martineau (Séminaire Bourbaki, 1968) and Morimoto (see "Microfunctions...", Lecture Notes in Math., Springer Verlag, 1971). 
that: $\sum_{j \in J} \tilde{f}_{j}=0$, there exists a set of distributions $\tilde{f}_{i j}=b . v \cdot F_{i j}$, analytic in $T_{B_{i j} \oplus \alpha}$, (where $\left.B_{i j}=\left(B_{i} \cup B_{j}\right)^{c}\right)$ such that:

$$
\begin{gathered}
\tilde{f}_{i j}=-\tilde{f}_{j i} \\
\tilde{f}_{j}=\sum_{i \neq j} \tilde{f}_{j i} .
\end{gathered}
$$

\section{§4. General Decomposability of the Essential Support $\mathrm{S}(\tilde{f})$ of a Distribution $\tilde{f}$.}

Using as basic tools the horizontal decomposability theorems stated in II, the microlocal decomposability of $S_{p}(\tilde{f})$ and corresponding generalized edge-of-the-wege theorems stated in III. 3, one can derive the following general decomposability property of $S(\tilde{f})$ :

Theorem. For every finite family of open sets $S_{i}(i \in I)$ in $T^{*} R$, the condition $S(\tilde{f}) \subset\left(\bigcup_{i \in I} S_{i}\right) \cup \mathscr{R}$ implies the existence of a (non unique) decomposition:

$$
\tilde{f}=\sum_{i \in I} \tilde{f}_{i}, \quad \text { with } \quad s\left(\tilde{f}_{i}\right) \subset S_{i} \cup \mathcal{R}
$$

Remark. The most interesting illustration of this theorem corresponds to the case when all $S_{i}$ are convex with respect to the fibers (more precisely when all their fibers in $T_{p} * \mathscr{R}$ are convex salient cones): in this case, one has $\tilde{f}_{i}=b \cdot v \cdot F_{i}$, where $F_{i}$ is analytic in a general tuboid whose profile $\Lambda_{i}$ in $T \mathscr{R}$ is easily determined from $S_{i}: \Lambda_{i}=\bigcup_{p}\left(p,\left(\Lambda_{i}\right)_{p}\right)$ with $\left(\Lambda_{i}\right)_{p}=\left(\widetilde{S}_{i}\right)_{p}$.

\section{Bibliography}

Concerning the boundary values of analytic functions in the sense of distributions, see the basic work of A. MARTINEAU: "Distributions et valeurs au bord des fonctions holomorphes" in the "Cours International d'été sur la théorie des destributions" -Lisbonne-Sept. 1964.

For a proof of the generalized Laplace transform theorem, in the case of $C^{\infty}$ boundary values see:-J. Bros and D. Iagolnitzer, Proceedings of the Marseille Conference on Renormalization theory (June 1971) and Ann. Inst. Poincaré vol. 18, $\mathrm{n}^{\circ} 2,147$ (1973).

The content of part II has been presented in detail in:-J. Bros and D. Iagolnitzer, Ann. Inst. Fourier 1976 and in the "Seminaire Goulaouic-Lions-Schwartz" Ecole Polytechnique-Paris 1974-1975: Exposé $n^{\circ} 16$ by J. Bros.

The microlocal developments of part III have been partly presented in the "Séminaire Goulaouic-Lions-Schwartz". Ecole Polytechnique-Paris 1974-75: Exposé ${ }^{\circ} 18$ by D. Iagolnitzer and in the Proceedings of Les Houches Summer School-June 1975.

References to related subjects and to known theorem have been given at the relevant places in the text. 
